\title{
Uncovering genetic and non-genetic biomarkers specific for exudative age-related macular degeneration: significant association of twelve variants
}

\author{
Raffaella Cascella1,2,\#, Claudia Strafella ${ }^{3,4, \#}$, Giuliana Longo ${ }^{3}$, Michele Ragazzo $^{1,5}$, \\ Laura Manzo ${ }^{3,4}$, Cecilia De Felici ${ }^{6}$, Valeria Errichiello ${ }^{3}$, Valerio Caputo ${ }^{3}$, Francesco \\ Viola7, Chiara Maria Eandi, Giovanni Staurenghi ${ }^{9}$, Andrea Cusumano ${ }^{6}$, Silvestro \\ Mauriello ${ }^{3}$, Luigi Tonino Marsella ${ }^{3}$, Cinzia Ciccacci ${ }^{3}$, Paola Borgiani ${ }^{3}$, Federica \\ Sangiuolo ${ }^{3}$, Giuseppe Novelli ${ }^{3}$, Federico Ricci, ${ }^{6, *}$ and Emiliano Giardina ${ }^{1,3, *}$ \\ ${ }^{1}$ Molecular Genetics Laboratory UILDM, Santa Lucia Foundation, Rome, Italy \\ ${ }^{2}$ Department of Chemical Pharmaceutical and Biomolecular Technologies, Catholic University Our Lady of Good Counsel, \\ Tirane, Albania \\ ${ }^{3}$ Department of Biomedicine and Prevention, Tor Vergata University, Rome, Italy \\ ${ }^{4}$ Emotest Laboratory, Pozzuoli, Italy \\ ${ }^{5}$ Department of Medical Science, Catholic University Our Lady of Good Counsel, Tirane, Albania \\ ${ }^{6}$ UOSD Retinal Pathology PTV Foundation Policlinico Tor Vergata, Rome, Italy \\ ${ }^{7}$ U.O. Oculist Foundation IRCCS Cà Granda Maggiore General Hospital, University of Milan, Milan, Italy \\ ${ }^{8}$ Department of Clinical Physiopathology, Eye Clinic, University of Turin, Turin, Italy \\ ${ }^{9}$ Eye Clinic, Department of Clinical Science Luigi Sacco, Luigi Sacco Hospital, University of Milan, Milan, Italy \\ \#The authors contributed equally to this manuscript \\ *These authors contributed equally to this work
}

Correspondence to: Raffaella Cascella, email: raffaellacascella@virgilio.it

Keywords: age related macular degeneration; macula; susceptibility; genomic and non-genomic factors

Received: October 05, $2017 \quad$ Accepted: December 01, $2017 \quad$ Published: December 12, 2017

Copyright: Cascella et al. This is an open-access article distributed under the terms of the Creative Commons Attribution License 3.0 (CC BY 3.0), which permits unrestricted use, distribution, and reproduction in any medium, provided the original author and source are credited.

\section{ABSTRACT}

Age-related Macular Degeneration (AMD) represents one of the most sightthreatening diseases in developed countries that substantially impacts the patients' lifestyle by compromising everyday activities, such as reading and driving. In this context, understanding the prevalence, burden, and population-specific risk/ protective factors of AMD is essential for adequate health care planning and provision. Our work aimed to characterize exudative AMD in Italian population and to identify the susceptibility/protective factors (genetic variants, age, sex, smoking and dietary habits) which are specific for the onset of disease. Our study involved a cohort of 1976 subjects, including 976 patients affected with exudative AMD and 1000 control subjects. In particular, the sample cohort has been subjected to a large genotyping analysis of $\mathbf{2 0}$ genetic variants which are known to be associated with AMD among European and Asiatic populations. This analysis revealed that 8 genetic variants (CFH, ARMS2, IL-8, TIMP3, SLC16A8, RAD51B, VEGFA and COL8A1) were significantly associated with AMD susceptibility. Successively, we performed a multivariate analysis, considering both genetic and non-genetic data available for our sample cohort. The multivariate analysis showed that age, smoking, dietary habits and sex, together with the genetic variants, were significantly associated with AMD in our population. Altogether, these data represent a starting point for the set-up of adequate preventive and personalized strategies aimed to decrease the burden of disease and improve the patients' quality of life. 


\section{INTRODUCTION}

AMD affects approximately 21 million people worldwide, and it is the main cause of low vision among people aged 55 years and older [1-2]. In Italy, over 1 million individuals manifest with initial symptoms of the disease, and 63000 new AMD cases are estimated each year [3]. The hallmarks of the disease include the progressive degeneration of the central part of the retina (macula) and drusen formation, changes that ultimately disrupt the cytoarchitectonics of the central retina and cause atrophy or abnormal choroidal neovascularization $(\mathrm{CNV})$. According to the clinical phenotype, AMD is classified as non-exudative or exudative [3-5]. In the early stage of disease, fundus examination reveals the presence of non-exudative lesions such as drusen, areas of Retinal Pigment Epithelium (RPE) depigmentation or pigment clumping. These drusen typically occur with significant pigment changes and accumulation of pigment in the posterior pole. Morphological characteristics of the drusen (size, coalescence, extension) and the occurrence of pigment changes appear to be correlated with the risk of disease evolution. The late stage of AMD is characterized by either the presence of central retinal atrophy, termed geographic atrophy (GA), or the presence of an exudative neovascular lesion, known as choroidal neovascularization (CNV) [5-6]. In the presence of CNV, the abnormal blood vessels typically sprout from the choriocapillaris and can penetrate Bruch's membrane (BrM) into the space beneath the RPE (type $1 \mathrm{CNV}$ ) or the subretinal space (type 2 $\mathrm{CNV}$ ). In some cases, neovascularization appears to initiate from the retinal vessels, induces detachment of the pigment epithelium and finally creates anastomosis with choroidal vasculature (Retinal Angiomatous ProliferationRAP or type $3 \mathrm{CNV}$ ).

Concerning the onset and the evolution of the disease, a number of triggering factors have been described up to date. First, aging is responsible for the loss of approximately $30 \%$ of rod photoreceptors and has been directly correlated with disease prevalence $[3-4,7-8]$. Second, smoking is known to significantly increase the risk for AMD as a result of the RPE damage caused by the release of oxidative compounds contained in cigarettes $[1,5,9-10]$. The damages caused by cigarettes smoke appeared to be strongly correlated with the exudative form of AMD. Third, dietary habits, especially the consumption of food with antioxidant properties, are usually associated with slower disease progression towards more severe forms $[1,5,11]$. In fact, a number of studies have demonstrated that patients treated with antioxidant nutrients (carotenoids, vitamins, mineral elements and Omega-3 fatty acids) have 25\% lower risk of progression towards the advanced stage of AMD and 19\% lower risk of vision loss [12]. Moreover, subjects experiencing a healthy lifestyle (meant as regular consumption of antioxidant nutrients, no smoking and physical activity) had 3-fold lower risk of early AMD compared to people having unhealthy lifestyle [13].

Furthermore, familiarity is another susceptibility factor, showing an estimated value of $11 \%$ in the presence of affected first-degree relatives [3, 14].

The contribution of genomics to AMD has been so far described through several common and rare variants mapped on different genes. Of these, the following are noteworthy: $C F H$ (rs1061170, T/C), ARMS2 (rs10490924, $\mathrm{G} / \mathrm{T}), \quad I L-8$ (rs2227306, C/T), C2 (rs547154, C/A; rs9332739, G/C), CFB (rs4151667, T/A), C3 (rs2230199, $\mathrm{C} / \mathrm{G}), C 9$ (rs62358361, G/T), CFI (rs4698775, G/T), TNFRSF10A (rs13278062, G/T), TIMP3 (rs5749482, $\mathrm{C} / \mathrm{G}), V E G F A$ (rs943080, C/T), COL8A1 (rs13081855, $\mathrm{G} / \mathrm{T}), \operatorname{SLC} 16 A 8$ (rs8135665, G/T), RAD51B (rs8017304, $\mathrm{A} / \mathrm{G}), \operatorname{ADAMTS} 9$ (rs679573, C/T), LIPC (rs920915, C/G), APOE (rs4420638, $\mathrm{A} / \mathrm{G}), C O L 10 A 1$ (rs3812111, $\mathrm{A} / \mathrm{T})$, IER3-DDR1 (rs1864163, A/G), B3GALTL (rs9542236, $\mathrm{C} / \mathrm{T}), T G F B R 1$ (rs334353, T/G), and CETP ( $\mathrm{rs} 1864163$, G/A) [5, 15-17]. Currently, only $C F H, A R M S 2$ and $I L-8$ are known to be associated with AMD in the Italian population [3, 18-20]. Therefore, we performed a large genotyping analysis to determine the genetic variations associated with Italian patients affected by AMD. Successively, we performed a multivariate analysis, taking into account all genetic data and non-genetic factors (age, sex, smoking and dietary habits). The ultimate goal of the multivariate analysis was to determine the contribution of genetic and environmental factors to the onset of AMD in Italian patients. Moreover, bioinformatic analysis predicted some possible gene interactions at the basis of AMD pathogenesis.

\section{RESULTS}

All of the 1976 samples were genotyped for the selected SNPs. Of the analyzed variants, only 8 SNPs yielded significant association values. As expected, we confirmed that rs 1061170 (CFH), rs10490924 (ARMS2), rs2227306 (IL-8) had a strong association with AMD susceptibility in the Italian population. In addition, the biostatistical analysis revealed the association of 5 new SNPs in our population: rs5749482 (TIMP3), rs8017304 (RAD51B), rs8135665 (SLC16A8), rs943080 (VEGFA), and rs13081855 (COL8A1). The other SNPs tested for statistical association did not yield significant values. The $p$ and ORs for the 20 SNPs are reported in Table 1.

The multivariate logistic regression analysis was performed accounting for the 8 associated SNPs, age, sex, smoking and dietary (consumption of fruits and vegetables) habits collected from our Italian subjects.

We performed 3 different levels of multivariate logistic regression analysis to estimate the contribution of genetic and non-genetic factors to AMD susceptibility.

The first level of the multivariate analysis included only the frequency distribution of the 8 associated SNPs. 
Table 1: Association analysis results of the 20 selected SNPs

\begin{tabular}{|c|c|c|c|c|c|}
\hline GENES & SNPs & LOCUS & $p$ & OR (CI 95\%) & EFFECT \\
\hline $\mathrm{CFH}$ & rs1061170 (T/C) & $1 \mathrm{q} 31$ & $1.05^{*} 10^{-34}$ & $\mathrm{C}=2.3(2.0-2.6)$ & risk \\
\hline ARMS2 & rs10490924 (G/T) & $10 q 26$ & $2.37^{*} 10^{-42}$ & $\mathrm{~T}=2.6(2.3-3.0)$ & risk \\
\hline IL8 & rs2227306 (C/T) & $4 q 13$ & $1.53^{*} 10^{-05}$ & $\mathrm{~T}=1.4(1.2-1.5)$ & risk \\
\hline TIMP3 & rs5749482 (C/G) & $22 q 12.3$ & $5.96^{*} 10^{-06}$ & $\mathrm{C}=1.6(1.3-1.9)$ & risk \\
\hline$L I P C$ & rs920915 (C/G) & $15 \mathrm{q} 21$ & ns & - & - \\
\hline APOE & rs4420638 (A/G) & $19 \mathrm{p} 13.2$ & ns & - & - \\
\hline VEGFA & rs943080 (C/T) & $6 \mathrm{p} 21.1$ & $1.13^{*} 10^{-04}$ & $\mathrm{~T}=1.3(1.1-1.4)$ & risk \\
\hline IER3-DDRI & rs1864163 (A/G) & $6 \mathrm{p} 21.3$ & $\mathrm{~ns}$ & - & - \\
\hline$B 3 G A L T L$ & rs9542236 (C/T) & $13 q 12.3$ & ns & - & - \\
\hline TGFBR1 & rs334353 (T/G) & $9 q 22$ & ns & - & - \\
\hline ADAMTS9 & rs679573 (C/T) & $3 p 14.1$ & ns & & - \\
\hline COL10A1 & rs3812111 (A/T) & $6 \mathrm{q} 21$ & ns & - & - \\
\hline CETP & rs1864163 (G/A) & $16 \mathrm{q} 21$ & $\mathrm{~ns}$ & - & - \\
\hline$R A D 51 B$ & rs8017304 (A/G) & $14 q 23$ & 0.007 & $\mathrm{G}=1.2(1.0-1.4)$ & risk \\
\hline$S L C 16 A 8$ & rs8135665 (C/T) & $22 q 13.1$ & $1.89^{*} 10^{-08}$ & $\mathrm{~T}=1.6(1.4-1.9)$ & risk \\
\hline COLSA1 & rs $13081855(\mathrm{G} / \mathrm{T})$ & $3 q 12.1$ & $1.02^{*} 10^{-08}$ & $\mathrm{~T}=2.0(1.5-2.5)$ & risk \\
\hline$C 2$ & $\begin{array}{l}\text { rs547154 (C/A) } \\
\text { rs9332739 (G/C) }\end{array}$ & $6 \mathrm{p} 21.3$ & ns & - & - \\
\hline$C F B$ & rs4151667 (T/A) & $6 \mathrm{p} 21.3$ & ns & - & - \\
\hline C3 & rs2230199 (C/G) & 19p13.3 & ns & - & - \\
\hline
\end{tabular}

Interestingly, the logistic regression coefficient $\left(\mathrm{R}^{2}\right)$ revealed that genetic factors account for $23 \%$ of the disease susceptibility. Moreover, the significant associations of CFH, ARMS2, IL-8, TIMP3, SLC16A8, VEGFA and COL8A1 were confirmed, while the association of $R A D 51 B$ was not significant. This result suggested that $R A D 51 B$ is a very low risk factor for AMD in the Italian population compared with stronger susceptibility genes.

The second level of the multivariate analysis was performed taking into account only the non-genetic factors, which were age, sex, smoking and dietary habits. The overall contribution of these variables to AMD susceptibility was estimated to be $10 \%\left(\mathrm{R}^{2}\right)$. In particular, advanced age and smoking status were strongly associated with a higher risk of AMD, while the regular consumption of fruits and vegetables showed to be protective from the disease. The association between sex and disease susceptibility was non-significant at this level of analysis. The $p$ and ORs for each non-genetic variable are reported in Table 2.

The third level of the multivariate analysis included all genetic and non-genetic factors taken into consideration in our work. The logistic regression coefficient $\left(\mathrm{R}^{2}\right)$ suggested that all of the tested variables were able to describe up to $29 \%$ of disease susceptibility. Overall, $C F H$ and $A R M S 2$ remained the most significant risk factors for AMD, while $R A D 51 B$ was identified as the least relevant gene. Surprisingly, at this step of the analysis, sex appeared to be slightly associated with the disease. In particular, females appeared to be at lower risk for AMD with respect to males. The $p$ and ORs for the genetic and non-genetic variables are reported in Table 3.

The bioinformatic analysis of the gene interactions through WEB-based GEne SeT AnaLysis Toolkit and DAVID Bioinformatic Database, provided different results depending on the selected enrichment tool (GO Analysis, Pathway Commons Analysis and Transcription Factor Target Analysis). With regard to biological processes, the GO Analysis revealed significant interactions for all the genes of interest (Figure 1). The Pathway Commons Analysis showed that VEGFA and $I L-8$ share different pathways ( $p$ ranging from 0.02 and 0.008 ), especially those involved in signaling, metabolism and regulation of immune and inflammatory events. In addition, $R A D 51 B$ and $S L C 16 A 8$ showed a significant interaction $(p=0.002)$ in the modulation of the haemostasis/coagulation pathway. Transcription Factor Target Analysis reported significant associations with some transcription factors (TF). Of these TFs, the most relevant target was NFAT, which was associated with VEGFA and COL8A1 $(p=0.004)$.

With regard to gene/phenotype interactions, analyses performed using SPSS software demonstrated an association between VEGFA and type $1 \mathrm{CNV}$, with $p=0.006$ and $\mathrm{OR}=1.97$ (95\% CI: $1.20-3.23)$; but no significant associations were observed for the type 2 CNV subtype. However, the other genes did not appear 
Table 2: Multivariate analysis performed on the non-genetic variables

\begin{tabular}{cccc}
\hline NON-GENETIC VARIABLES & $\boldsymbol{p}$ & OR (CI 95\%) & EFFECT \\
Age & $1.58^{*} 10^{-36}$ & $1.4(1.3-1.5)$ & risk \\
Smoking habit & $1.31^{*} 10^{-4}$ & $1.4(1.2-1.7)$ & risk \\
Dietary habits (fruits\&vegetables) & $1.43^{*} 10^{-4}$ & $0.6(0.5-0.8)$ & protective \\
Sex & ns & - & - \\
\hline
\end{tabular}

to affect a specific subtype of exudative AMD, rather they should be regarded as susceptibility genes regardless of the disease phenotype.

\section{DISCUSSION}

The contribution of genomics to the risk of developing AMD has been extensively explored among global populations, particularly in European and Asian groups. According to recent data and disease prevalence estimates, up to 34 loci are known to account for approximately $46-71 \%$ of the risk of AMD globally $[1,17]$.

This study was designed to assess the specific contribution of genetic and environmental factors to the onset of exudative AMD in Italian population. To this end, 1976 Italian subjects were genotyped for 20 variants, which have previously been associated with the disease in European and Asiatic populations. Given our results, only 8 genetic variants (CFH, ARMS2, IL-8, TIMP3, SLC16A8, RAD51B, VEGFA and COL8A1) out of 20 reached the significance threshold fixed at $p<0.05$. As expected, $C F H$ and ARMS2 appeared to be the most associated with AMD. The association between $I L-8$ and the disease was confirmed in our cohort, although it has never been tested in other populations.

According to multivariate logistic regression analysis, the identified genetic factors were estimated to account for $23 \%$ of AMD susceptibility in Italian cohort. It is important to note that this estimate accounted for all of the associated genes except for RAD51B. Thus, $R A D 51 B$ can be considered a very low risk factor for
AMD, and it actually loses its significance when taken together with more strongly associated genes $(C F H$, ARMS2, IL-8, TIMP3, SLC16A8, VEGFA and COL8A1). Moreover, the multivariate logistic regression analysis reported that $C F H$ and $A R M S 2$ accounted for $20 \%$ of the overall genetic susceptibility to AMD, while the remaining $3 \%$ was attributable to $I L-8, T I M P 3$, SLC16A8, VEGFA and COL8A1. Next, we evaluated the distribution of the number of risk alleles between cases and controls. As expected, the frequency distribution in the two groups showed different trends (Figure 2A), with a higher number of risk alleles in cases (Figure 2B) with respect to control subjects (Figure 2C). In fact, considering 7 as the median point out of 14 possible variant combinations, we observed a higher number of cases with at least 7 risk alleles compared with the control group. Thus, the presence of an increased number of risk alleles in AMD patients allowed us to presume a cumulative effect of genetic variants in determining the disease phenotype.

The secondary goal of the study was to estimate the contribution of non-genetic factors to the risk of AMD. To this end, we decided to consider environmental variables (age, sex, smoking and dietary habits) that are known to strongly impact the onset/progression of disease. As expected, advanced age and smoking status were found to be strongly associated risk factors for AMD, while regular consumption of fruits and vegetables represented a helpful source for preventing disease onset/progression. However, our multivariate analysis showed that non-genetic factors accounted for $10 \%$ of disease, in contrast with the $19-37 \%$ described in the literature [1].

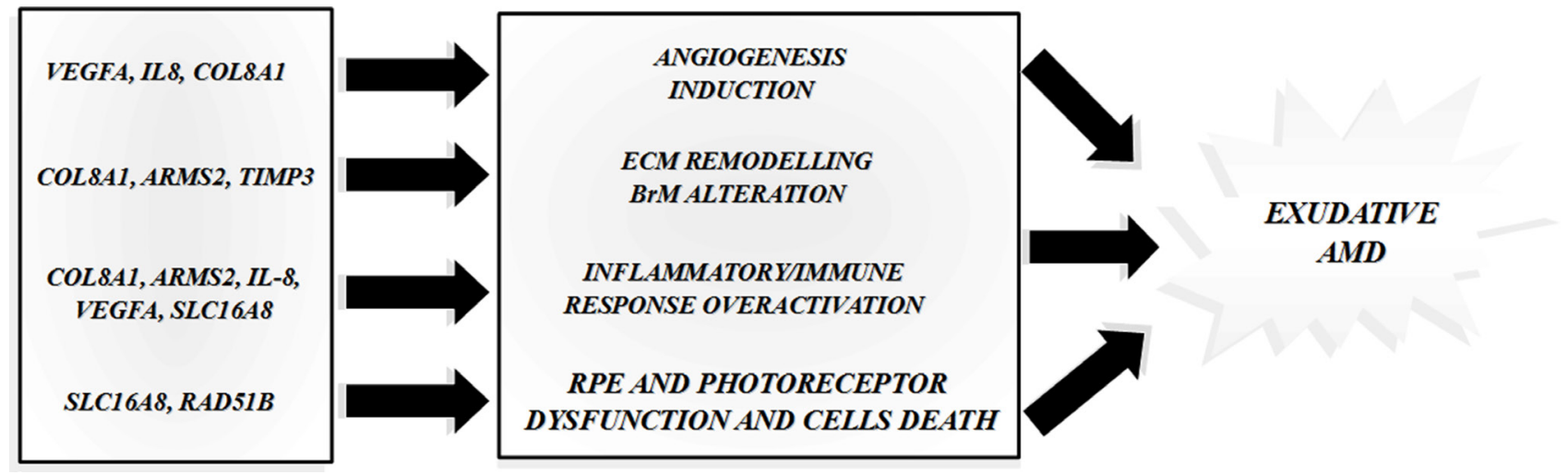

Figure 1: Predicted interactions among the AMD-associated genes. The figure shows the main gene interactions derived by the prediction analysis with the bioinformatic tools. 
Table 3: Multivariate analysis performed on genetic and non-genetic variables

\begin{tabular}{|c|c|c|c|}
\hline CONSIDERED VARIABLES & $p$ & OR (CI 95\%) & EFFECT \\
\hline $\mathrm{CFH}(\mathrm{rs1061170}, \mathrm{T} / \mathrm{C})$ & $1.29^{*} 10^{-23}$ & $\mathrm{C}=2.7(2.2-3.3)$ & risk \\
\hline$A R M S 2(\mathrm{rs} 10490924, \mathrm{G} / \mathrm{T})$ & $2.19^{*} 10^{-26}$ & $\mathrm{~T}=3.1(2.5-3.9)$ & risk \\
\hline IL8 (rs2227306, C/T) & $2^{*} 10^{-4}$ & $\mathrm{~T}=1.4(1.1-1.7)$ & risk \\
\hline TIMP3 (rs5749482, C/G) & $4^{*} 10^{-4}$ & $\mathrm{C}=1.7(1.5-2.2)$ & risk \\
\hline$V E G F A(\mathrm{rs} 943080, \mathrm{C} / \mathrm{T})$ & 0.032 & $\mathrm{~T}=1.2(1.0-1.5)$ & risk \\
\hline$R A D 51 B(\mathrm{rs8017304,} \mathrm{A} / \mathrm{G})$ & ns & - & - \\
\hline $\operatorname{SLC16A8}(\mathrm{rs8135665}, \mathrm{C} / \mathrm{T})$ & $5.71^{*} 10^{-11}$ & $\mathrm{~T}=2.1(1.7-2.7)$ & risk \\
\hline COL8A1 (rs13081855, G/T) & 0.014 & $\mathrm{~T}=1.5(1.1-2.1)$ & risk \\
\hline Age & $2.48^{*} 10^{-19}$ & $1.4(1.3-1.5)$ & risk \\
\hline Smoking habit & $7.85^{*} 10^{-5}$ & $1.7(1.3-2.2)$ & risk \\
\hline Dietary habits (fruits\&vegetables) & 0.015 & $0.6(0.4-0.9)$ & protective \\
\hline Female sex & 0.04 & $0.7(0.5-0.9)$ & protective \\
\hline
\end{tabular}

Afterwards, genetic and non-genetic factors were combined in one multivariate analysis, in order to provide a comprehensive overview of AMD in Italian population. Our results suggested that all tested variants were able to cover $29 \%$ of disease. In particular, genes $(C F H$, ARMS2, IL-8, TIMP3, SLC16A8, VEGFA and COL8A1), old age and smoking status contribute to susceptibility, while female sex and regular consumption of fruits and vegetables protect from AMD development.

Interestingly, our results highlighted a prominent difference between the Italian and global population, in which the genetic contribution has been estimated to range from $46 \%$ to $71 \%$ [1]. These data suggest that Italianassociated genes may have a greater impact on disease in our population. In fact, these genes are sufficient to account for $23 \%$ of susceptibility, in contrast to the 34 loci required to account for $46-71 \%$ of AMD risk across the global populations. Overall, our results suggest that AMD onset in the Italian population may be regulated by distinct interaction patterns involving few genes. To this end, we explored potential interactions of the associated genes and their function. In particular, $\mathrm{CFH}$ functions as an essential
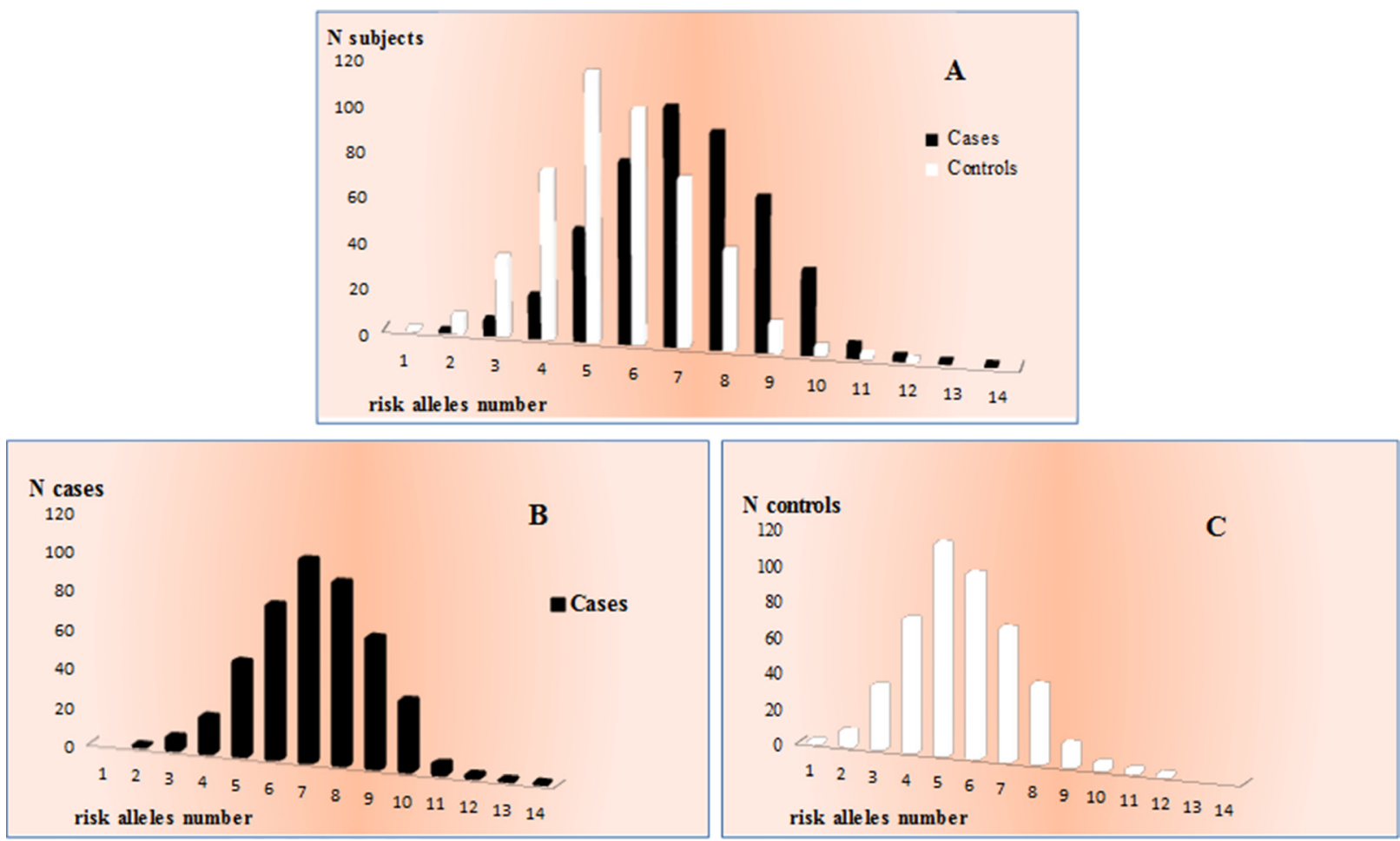

Figure 2: Distribution of the number of risk alleles between cases and controls. (A) allele frequency distribution in the two groups. (B) allele frequency distribution in cases. (C) allele frequency distribution in control subjects. 
inhibitor of complement activation, and the homozygous genotype for the rs1061170 risk allele has been shown to increase the activation of the complement cascade [4-5, 21-22]. Although the role of ARMS2 needs to be clarified, studies performed on animal models have reported that it may be involved in angiogenesis, extracellular matrix mineralization, and transforming growth factor-beta signaling [4, 23-24].

In addition, ARMS2 may interact with proinflammatory molecules, such as IL-6, IL- 8 , TNF- $\alpha$, C3 and C5 [23]. IL-8 is known to be a primary mediator of angiogenesis, and it is fundamentally involved in the chemotaxis of immune cells (neutrophils and lymphocytes) to the site of inflammation [20]. Regarding the activation of angiogenesis, VEGFA is recognized as one of the most important growth factors triggering this mechanism $[1,5]$. Therefore, it is not surprising that our bioinformatic analysis suggested significant possible interactions between $I L-8, V E G F A, C F H$ and $A R M S 2$. Thus, the involvement of $C F H, A R M S 2, I L-8$ and $V E G F A$ in the inflammatory, angiogenic and tissue homoeostasis pathways fits perfectly with the clinical phenotype displayed by patients affected with exudative AMD. In addition, the gene/phenotype interaction analysis revealed that the risk allele (T) of VEGFA (rs943080) is more frequent among patients with type $1 \mathrm{CNV}$ compared with those with the type $2 \mathrm{CNV}$ subtype. Importantly, rs943080 maps to a highly evolutionary conserved region of chromosome 6 . In particular, the alternative allele $\mathrm{C}$ is believed to disrupt the binding site of conerod homeobox (CRX), which is an essential transcription factor that is highly expressed in RPE and retinal ganglion cells [25-26]. Thus, the disruption of the CRX binding site may reduce the expression of VEGFA beneath the RPE monolayer and decrease neovascularization in type 1 exudative AMD. In study involving 223 patients with neovascular AMD, subjects carrying the VEGFA rs 943080 risk genotype (TT) had 1.8-fold higher expression of $V E G F A$ compared with patients with the protective $V E G F A$ rs943080 genotype (CC) variant $(p=0.012)$ [26]. Based on these data, VEGFA genotyping may help clinicians to recognize some clinical features indicative of type 1 exudative AMD. Moreover, the TT genotype of VEGFA rs 943080 has been associated with a poorer response to anti-VEGFA therapy compared with the low-risk genotype (CC) [26-27]. These data indicate VEGFA may be a pharmacogenetic biomarker that influences the degree of response to anti-VEGFA treatments; the biomarker may be useful to determine the dosage and administration frequency according to the patient's genotype.

Interestingly, the bioinformatic analysis showed a significant interaction between VEGFA and COL8A1. The homonymous protein encoded by COL8A1 is a major component of Descemet's membrane of corneal and blood vessel endothelial cells. COL8A1 permits the migration and proliferation of vascular smooth muscle cells, is involved in the maintenance of vessel wall integrity and structure and participates in extracellular matrix (ECM) remodeling and angiogenesis [4-5, 17]. We therefore believe that genetic variants in COL8A1 may result in altered protein, causing more fragile vessels, vessel disruption and the onset of edema that is normally associated with wet AMD. Another possible role of COL8A1 in AMD pathogenesis may lie in its participation in ECM remodeling pathways, which thereby trigger the progression of angiogenesis.

Moreover, the TF target bioinformatic analysis revealed a significant relationship between COL8A1 and $V E G F A$ with NFAT transcription factors. The NFAT TFs are a family of proteins mainly involved in immune responses and VEGF-induced angiogenesis and therefore have a potential role in the aetiopathogenesis of AMD [28].

Regarding interactions among TIMP3, VEGFA, $R A D 51 B$ and SLC16A8, bioinformatic data indicated significant interactions only for RAD51B, VEGFA and SLC16A8. In particular, TIMP3 is an inhibitor of metalloproteinase 3, which is involved in the degradation of extracellular matrix. It is present in BrM, where it is essential for ECM maintenance and remodeling [4, $6,17]$. In this context, AMD-associated variations in ECM proteins can alter the structure and permeability of the BrM, making it more susceptible to aging and environmental abuse. $R A D 51 B$ is essential for the activation of DNA-repair mechanisms and may play a role in the induction of RPE and the photoreceptor cell death at the root of AMD pathogenesis [5]. SLC16A8 encodes a transporter protein comprising many shortchain monocarboxylates that spans the membrane. This protein is specifically expressed by the RPE and a deficit in lactate transport may cause the retinal acidification and photoreceptor dysfunction [17]. Altogether, our bioinformatic results provided interesting insights into the interactions among AMD-associated genes, which may thereby trigger alterations of BrM structure and permeability, the induction of angiogenesis and the damage caused by aging and environmental factors. Clearly, further molecular and cellular experiments are necessary to verify the effective relationships between CFH, ARMS2, IL-8, TIMP3, SLC16A8, VEGFA and $C O L 8 A 1$ in the development and progression of the disease.

Our work provides a general overview of the genetic and non-genetic factors (which contribute to the onset of exudative AMD in Italian population. The extensive analysis performed on 1976 subjects highlighted 8 genetic variants and 4 non-genetic factors associated with exudative AMD.

Regarding genetic susceptibility, our findings differed from global studies that have described many more associated variants than the 8 reported above. In addition, the bioinformatic analysis revealed interesting 
insights into the biological functions and interactions among the genes associated with AMD in our cohort. Our findings suggest that $C F H, A R M S 2, I L-8$, TIMP3, SLC16A8, RAD51B, VEGFA and COL8A1 may contribute to the onset of wet AMD at different levels, particularly the following: induction of angiogenesis; alteration of ECM remodeling mechanisms and of BrM integrity and permeability; modification of RPE and photoreceptor cell activities; and over-activation of inflammatory and immune responses.

Regarding environmental effects on the disease, our data confirmed previously reported findings. As expected, smoking status and advanced age were two of the major risk factors for AMD, while regular consumption of fruits and vegetables was as preventative factor. On the other hand, the association between sex and AMD was discordant with previous data. In our study, women had a lower risk of disease, while other studies have reported non-significant or conflicting data on this subject. However, the association value was very weak in our study, and may not be relevant in comparison with other environmental factors. Overall, our study showed that AMD susceptibility within the Italian population is contributed to by genetic variants, accounting for $23 \%$ of disease and non-genetic variants, accounting for $10 \%$ of AMD (Figure 3). The remaining $67 \%$ of disease susceptibility is still a matter of investigation. Other promising biomarkers certainly contribute to disease, among which epigenetic factors are of great interest. In this context, special attention should be paid to methylation patterns and miRNAs, since they are likely to be involved in the mechanisms underlying the onset and progression of disease. In particular, methylation seems to impact the proinflammatory activity and oxidative response which are significantly increased in AMD pathogenesis [1, 29]. MiRNAs have been closely connected with angiogenic, inflammatory and cell survival mechanisms which are usually disrupted in case of disease [5, 29]. Although the relationship with AMD needs to be clarified, epigenetic modifications represent the most promising biomarkers for the prediction of the risk/ protection to exudative AMD as well as for the development of new drugs.

\section{MATERIALS AND METHODS}

\section{Study cohort and DNA extraction}

This study enrolled 1976 individuals, including 976 wet-AMD cases and 1000 control subjects. Cases were recruited from the Ophthalmology Unit of the PTV General Hospital of Rome, U.O. Oculist Foundation IRCCS "Cà Granda" Maggiore General Hospital of Milan, Department of Clinical Physiopathology of the University of Turin, Department of Clinical Science of Sacco Hospital, of Milan. Patients were selected following specific inclusion criteria: male or female subjects over the age of 50 years, diagnosis of exudative AMD, clear media in order to guarantee good quality images. The exclusion criteria included: signs of any other macular condition predisposing to $\mathrm{CNV}$, any previous intraocular treatment, including laser photocoagulation and photodynamic therapy with verteporfin (PDT), cataract surgery, allergy to fluorescein. The control subjects were recruited from the UOSD SIMT and the Ophthalmology Unit of the PTV General Hospital of Rome. Recruited subjects were asked to reply to a questionnaire in order to classify their smoking and dietary habits. Subjects who have never smoked or quit smoking $\geq 5$ years before recruitment were

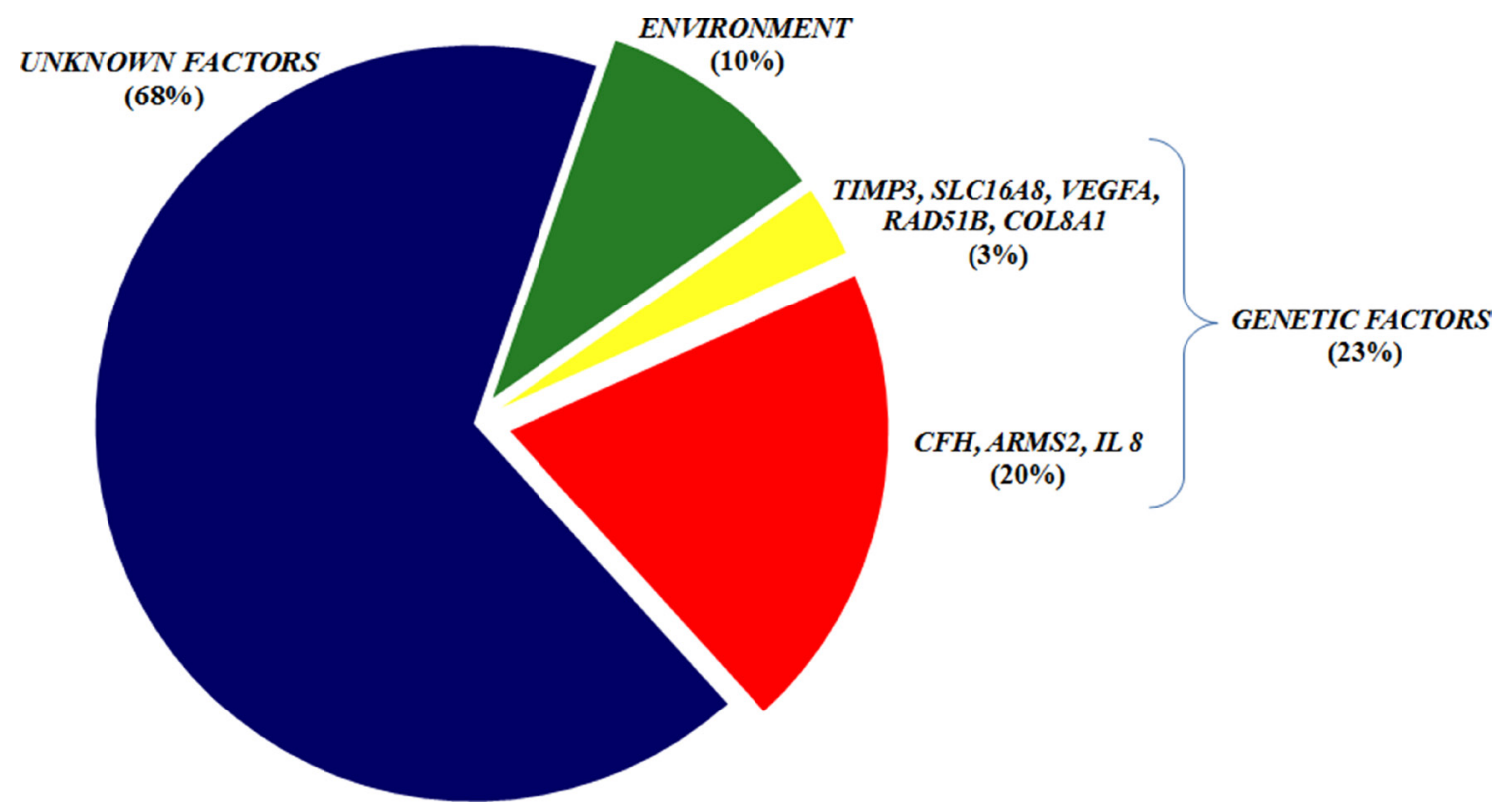

Figure 3: The AMD susceptibility within the Italian population. The chart illustrates the main components able to describe the genetic and non-genetic variables contributing to the disease susceptibility in our population. 
Table 4: Collection of data concerning the subjects enrolled in the study

\begin{tabular}{ccc}
\hline DATA & CASES & CONTROLS \\
\hline Age & \pm 77 years old & \pm 72 years old \\
Sex & F: $54 \%$ & F: $56 \%$ \\
& M: $46 \%$ & M: $44 \%$ \\
Type of CNV & Type $1: 53 \%$ & - \\
& Type $2: 47 \%$ & R: $79 \%$ \\
Dietary habits & R: $86 \%$ & S: $21 \%$ \\
& S: $14 \%$ & Y: $47 \%$ \\
Smoking habit & Y: $47 \%$ & N: $53 \%$ \\
\hline
\end{tabular}

Concerning smoking habits, non-smokers status was assigned to those subjects who never smoked or quit smoking $\geq 5$ years before recruitment, otherwise they were considered as smokers. Dietary habits were sorted according to the frequency of fruits and vegetables intake ( $\geq 3$ times/week for regular consumption and $\leq 2$ times/week for unusual consumption). Legend: $\mathrm{F}=$ female; M: male; R: regular; S: seldom; Y: yes; N: not.

classified as non-smokers, otherwise they were considered as smokers. Dietary habits were sorted into regular intake of fruit and vegetables ( $\geq 3$ times per week) and unusual assumption ( $\leq 2$ times per week). All of the data related to the characterization of the study cohort are summarized in Table 4.

The study was approved by the ethics committee of the University of Rome "Tor Vergata" and was performed according to the Declaration of Helsinki. All participants provided signed informed consent. Blood samples were obtained from all subjects in order to extract genomic DNA. Genomic DNA extraction was performed using the EZ1 Advanced XL automated extractor and the EZ1 DNA Blood $200 \mu 1$ Kit (Qiagen).

\section{Genotyping analysis}

All of the samples (1976 individuals) were genotyped for the 22 selected SNPs, which were as follows: CFH (rs1061170, T/C), ARMS2 (rs10490924, $\mathrm{G} / \mathrm{T}), \quad I L-8 \quad(\mathrm{rs} 2227306, \mathrm{C} / \mathrm{T}), \quad C 2 \quad(\mathrm{rs} 547154, \mathrm{C} / \mathrm{A}$; rs9332739, G/C), CFB (rs4151667, T/A), C3 (rs2230199, $\mathrm{C} / \mathrm{G}), C F I$ (rs4698775, G/T), TNFRSF10A (rs13278062, G/T), TIMP3 (rs5749482, C/G), VEGFA (rs943080, C/T), COL8A1 (rs13081855, G/T), SLC16A8 (rs8135665, G/T), RAD51B (rs8017304, A/G), ADAM (rs679573, C/T), LIPC (rs920915, C/G), APOE (rs4420638, A/G), COL10A1 (rs3812111, A/T), IER3-DDR1 (rs1864163, A/G), B3GALTL (rs9542236, C/T), TGFBR1 (rs334353, T/G), and CETP (rs1864163, G/A). The CFI and TNFRSF10A genotype analyses were limited by technical issues, and therefore the required molecular experiments were not successfully completed.

All samples were genotyped using the TaqMan assay on a 7500 Fast Real Time PCR device according to the manufacturer's instructions (Applied Biosystems). The genotyping results were interpreted using Sequence Detection System 2.1 software (Applied Biosystems).
Each Real Time PCR run was performed using a negative control and three positive control samples previously confirmed by direct sequencing (BigDye Terminator v3.1, BigDyeXTerminator) on ABI3130xl (Applied Biosystems).

\section{Biostatistical and bioinformatic analysis}

The genotyping results were subjected to biostatistical analysis to evaluate associations with AMD. First, the genotyping data reported in our cohort were tested to confirm the Hardy-Weinberg equilibrium $(p>0.05)$. Afterwards, the association of the genotyped SNPs were measured by calculating the $p$-value $(p)$ through $2 \times 2$ contingency tables (http://www.physics. csbsju.edu/stats/contingency_NROW_NCOLUMN

form.html). The statistical associations were considered significant when $p<0.05$ based on the $95 \%$ confidence interval. The strength of the associations was determined by calculating the Odd Ratio (OR, http://www.hutchon. net/ConfidOR.htm).

The overall contribution of each genetic and nongenetic factor to AMD susceptibility was evaluated through a multivariate logistic regression analysis (stepwise method). The presence/absence of AMD was considered the dependent variable. First, the contributions of genetic factors associated with AMD in our cohort were determined. To this end, the analysis included $\mathrm{CFH}$, ARMS2, IL-8, TIMP3, COL8A1, SLC16A8, VEGFA, and $R A D 51 B$ as independent variables. Second, the contribution of non-genetic factors was estimated, taking into account age, sex, smoking and dietary habits as independent variables. The last analysis was performed considering both genetic and non-genetic factors as independent variables. At each level of the multivariate logistic regression analysis, the $\mathrm{R}^{2}$ (logistic regression coefficient) indicated the contribution of the considered variables to AMD susceptibility. The cut-off for statistical 
significance was set at a $p<0.05$. The multivariate analysis was performed using the SPSS program, ver. 19 (IBM Corp, Armonk, NY, USA).

\section{Bioinformatic evaluation of genes interactions}

All the associated genes were subjected to a bioinformatic analysis aimed to evaluate the potential presence of specific interactions among them. To this end, the WEB-based GEne SeT AnaLysis Toolkit [30] and DAVID Bioinformatic Database version 6.8 Beta [31-32] were utilized. The software incorporates information from different public resources and provides information about the possible biological and molecular pathways involving the genes of interest. The analysis was performed according to the following enrichment tools: GO Analysis, Pathway Commons Analysis and Transcription Factor Target Analysis. The results were considered significant with a cut-off of $p<0.05$.

\section{Abbreviations}

AMD: Age-related Macular Degeneration; SNPs: Single Nucleotide Polymorphisms; CNV: Choroidal NeoVascularization: RPE: Retinal Pigment Epithelium; GA: Geographic Atrophy; BrM: Bruch's membrane; TF: Transcription Factors; CRX: Cone-Rod homeoboX; $p$ : $p$-value; OR: Odd Ratio.

\section{Author contributions}

FR, AC, EG, made substantial contributions to conception and design of the study. RC, CS, GL, MR, $\mathrm{LM}, \mathrm{CC}, \mathrm{CDF}, \mathrm{VE}, \mathrm{VC}$ made substantial contributions to acquisition of data, analysis and interpretation of data. $\mathrm{RC}, \mathrm{CS}, \mathrm{GL}, \mathrm{CC}, \mathrm{FR}, \mathrm{EG}$ have been involved in drafting the manuscript. PB, AC, FR, GN, EG revising it critically for important intellectual content. RC, CS, MR, LM, GL, FC, CME, GS, SM, LTM, FS, PB, GN, AC, FR, EG have given final approval of the version to be published. FS, PB, GN, FR, EG are agreed to be accountable for all aspects of the work in ensuring that questions related to the accuracy or integrity of any part of the work are appropriately investigated and resolved.

\section{ACKNOWLEDGMENTS}

This work was kindly supported by Macula Foundation Onlus and the National Health Ministry (General Board of Medical Devices and of Pharmaceutical Service).

\section{CONFLICTS OF INTEREST} interests.

\section{FUNDING}

The present work has been founded by the National Health Ministry (General Board of Medical Devices and of Pharmaceutical Service, grant number: E82F16003700001).

\section{REFERENCES}

1. Sobrin L, Seddon JM. Nature and nurture- genes and environment- predict onset and progression of macular degeneration. Prog Retin Eye Res. 2014; 40:1-15.

2. Wong WL, Su X, Li X, Cheung CM, Klein R, Cheng CY, Wong TY. Global prevalence of age-related macular degeneration and disease burden projection for 2020 and 2040: a systematic review and meta-analysis. Lancet Glob Health. 2014; 2:e106-16.

3. Cascella R, Ragazzo M, Strafella C, Missiroli F, Borgiani P, Angelucci F, Marsella LT, Cusumano A, Novelli G, Ricci F, Giardina E. Age-related macular degeneration: insights into inflammatory genes. J Ophthalmol. 2014; 2014:582842.

4. Fritsche LG, Fariss RN, Stambolian D, Abecasis GR, Curcio CA, Swaroop A. Age-related macular degeneration: genetics and biology coming together. Annu Rev Genomics Hum Genet. 2014; 15:151-171.

5. Lambert NG, ElShelmani H, Singh MK, Mansergh FC, Wride MA, Padilla M, Keegan D, Hogg RE, Ambati BK. Risk factors and biomarkers of age-related macular degeneration. Prog Retin and Eye Res. 2016; 54:64-102.

6. Horie-Inoue K, Inoue S. Genomic aspects of age-related macular degeneration. Biochem Biophys Res Commun. 2014; 452:263-275.

7. Ehrlich R, Harris A, Kheradiya NS, Winston DM, Ciulla TA, Wirostko B. Age-related macular degeneration and the aging eye. Clin Interv Aging. 2008; 3:473-482.

8. Marazita MC, Dugour A, Marquioni-Ramella MD, Figueroa JM, Suburo AM. Oxidative stress-induced premature senescence dysregulates VEGF and $\mathrm{CFH}$ expression in retinal pigment epithelial cells: Implications for Age-related Macular Degeneration. Redox Biol. 2016; 7:78-87.

9. Hughes AE, Orr N, Patterson C, Esfandiary H, Hogg R, McConnell V, Silvestri G, Chakravarthy U. Neovascular agerelated macular degeneration risk based on CFH, LOC387715/ HTRA1, and smoking. PLoS Med. 2007; 4:e355.

10. Chen Y, Bedell M, Zhang K. Age-related macular degeneration: genetic and environmental factors of disease. Mol Interv. 2010; 10:271-281.

11. Zampatti S, Ricci F, Cusumano A, Marsella LT, Novelli G, Giardina E. Review of nutrient actions on age-related macular degeneration. Nutr Res. 2014; 34:95-105.

12. Awh CC, Lane AM, Hawken S, Zanke B, Kim IK. CFH and ARMS2 genetic polymorphisms predict response to antioxidants and zinc in patients with age-related macular degeneration. Ophthalmology. 2013; 120:2317-2323. 
13. Meyers KJ, Liu Z, Millen AE, Iyengar SK, Blodi BA, Johnson E, Snodderly DM, Klein ML, Gehrs KM, Tinker L, Sarto GE, Robinson J, Wallace RB, Mares JA. Joint Associations of Diet, Lifestyle, and Genes with AgeRelated Macular Degeneration. Ophthalmology. 2015; 122:2286-2294.

14. Wray NR, Yang J, Goddard ME, Visscher PM. The genetic interpretation of area under the ROC curve in genomic profiling. PLoS Genet. 2010; 6:e1000864.

15. Chen W, Stambolian D, Edwards AO, Branham KE, Othman M, Jakobsdottir J, Tosakulwong N, Pericak-Vance MA, Campochiaro PA, Klein ML, Tan PL, Conley YP, Kanda A, et al. Genetic variants near TIMP3 and highdensity lipoprotein-associated loci influence susceptibility to age-related macular degeneration. Proc Natl Acad Sci U.S.A. 2010; 107:7401e7406.

16. Fritsche LG, Chen W, Schu M, Yaspan BL, Yu Y, Thorleifsson G, Zack DJ, Arakawa S, Cipriani V, Ripke S, Igo RP Jr, Buitendijk GH, Sim X, et al. Seven new loci associated with age-related macular degeneration. Nat Genet. 2013; 45:433-439,439e1-2.

17. Fritsche LG, Igl W, Bailey JN, Grassmann F, Sengupta S, Bragg-Gresham JL, Burdon KP, Hebbring SJ, Wen C, Gorski M, Kim IK, Cho D, Zack D, et al. A large genomewide association study of age-related macular degeneration highlights contributions of rare and common variants. Nat Genet. 2016; 48:134e143.

18. Ricci F, Zampatti S, D’Abbruzzi F, Missiroli F, Martone C, Lepre T, Pietrangeli I, Sinibaldi C, Peconi C, Novelli $\mathrm{G}$, Giardina E. Typing of ARMS2 and CFH in age-related macular degeneration: case-control study and assessment of frequency in the Italian population. Arch Ophthalmol. 2009; 127:1368-1372.

19. Lepre T, Cascella R, Missiroli F, De Felici C, Taglia F, Zampatti S, Cusumano A, Ricci F, Giardina E, Eandi CM, Novelli G. Polymorphisms in ARMS2 (LOC387715) and LOXL1 Genes in the Japanese With Age-Related Macular Degeneration. Am J Ophthalmol. 2011; 152:325-326.

20. Ricci F, Staurenghi G, Lepre T, Missiroli F, Zampatti S, Cascella R, Borgiani P, Marsella LT, Eandi CM, Cusumano A, Novelli G, Giardina E. Haplotypes in IL-8 Gene Are Associated to Age-Related Macular Degeneration: A CaseControl Study. PLoS One. 2013; 8:e66978.

21. Van Lookeren Campagne M, Strauss EC, Yaspan BL. Age-related macular degeneration: Complement in action. Immunobiology. 2016; 221:733-739.
22. Schramm EC, Clark SJ, Triebwasser MP, Raychaudhuri S, Seddon JM, Atkinson JP. Genetic variants in the complement system predisposing to age-related macular degeneration: a review. Mol Immunol. 2014; 61:118-125.

23. Zeng F, Zhang M, Xu Y, Xu H. ARMS2 interference leads to decrease of proinflammatory mediators. Arch Clin Exp Ophthalmol. 2013; 251:2539-2544.

24. Fritsche LG, Loenhardt T, Janssen A, Fisher SA, Rivera A, Keilhauer $\mathrm{CN}$, Weber BH. Age-related macular degeneration is associated with an unstable ARMS2 (LOC387715) mRNA. Nat Genet. 2008; 40:892-896.

25. Yu Y, Bhangale TR, Fagerness J, Ripke S, Thorleifsson G, Tan PL, Souied EH, Richardson AJ, Merriam JE, Buitendijk GH, Reynolds R, Raychaudhuri S, Chin KA, et al. Common variants near FRK/COL10A1 and VEGFA are associated with advanced age-related macular degeneration. Hum Mol Genet. 2011; 20:3699-3709.

26. Zhao L, Grob S, Avery R, Kimura A, Pieramici D, Lee J, Rabena M, Ortiz S, Quach J, Cao G, Luo H, Zhang M, Pei M, et al. Common Variant in VEGFA and Response to Anti-VEGF Therapy for Neovascular Age-Related Macular Degeneration. Curr Mol Med. 2013; 13:929-934.

27. Dedania VS, Grob S. Pharmacogenomics of response to anti-VEGF therapy in exudative age-related macular degeneration. Retina. 2015; 35:381-391.

28. Zaichuk TA, Shroff EH, Emmanuel R, Filleur S, Nelius T, Volpert OV. Nuclear factor of activated T cells balances angiogenesis activation and inhibition. J Exp Med. 2004; 199:1513-1522.

29. Gemenetzi M, Lotery AJ. The role of epigenetics in agerelated macular degeneration. Eye. 2014; 28:1407-1417.

30. Wang J, Duncan D, Shi Z, Zhang B. WEB-based GEne SeT AnaLysis Toolkit (WebGestalt): update 2013. Nucleic Acids Res. 2013; 41:W77-83.

31. Huang DW, Sherman BT, Lempicki RA. Systematic and integrative analysis of large gene lists using DAVID Bioinformatics Resources. Nature Protoc. 2009; 4:44-57.

32. Huang DW, Sherman BT, Lempicki RA. Bioinformatics enrichment tools: paths toward the comprehensive functional analysis of large gene lists. Nucleic Acids Res. $2009 ; 37: 1-13$. 